\title{
Novel approach to diagnosis of His bundle capture using individualized left ventricular lateral wall activation time as reference
}

\author{
Marek Jastrzebski ${ }^{1}$, Pawel Moskal ${ }^{1}$, Piotr Kukla ${ }^{2}$, Agnieszka Bednarek ${ }^{1}$, Grzegorz \\ Kielbasa $^{1}$, Marek Rajzer ${ }^{1}$, Karol Curila ${ }^{3}$, and Pugazhendhi Vijayaraman ${ }^{4}$ \\ ${ }^{1}$ Jagiellonian University Collegium Medicum \\ ${ }^{2}$ Specialistic Hospital \\ 3, Charles University and University Hospital Kralovske Vinohrady \\ ${ }^{4}$ Geisinger Heart institute
}

June 29, 2021

\begin{abstract}
Background: During non-selective His bundle (HB) pacing, it is clinically important to confirm His bundle capture vs. right ventricular septal (RVS) capture. The present study aimed to validate the hypothesis that during HB capture left ventricular lateral wall activation time, approximated by the V6 R-wave peak time (V6RWPT), will not be longer than the corresponding activation time during native conduction. Methods: Consecutive patients with permanent HB pacing were recruited; cases with abnormal His-ventricle interval or left bundle branch block were excluded. Two corresponding intervals were compared: stimulus-V6RWPT and native HBpotential-V6RWPT. Difference between these two intervals (delta V6RWPT), diagnostic of lack of HB capture, was identified using receiver operating characteristic (ROC) curve analysis. Results: A total of 723 ECGs (219 with native rhythm, 172 with selective HB, 215 with non-selective HB, and 117 with RVS capture) were obtained from 219 patients. The native HB-V6RWPT, non-selective-, and selective-HB paced V6RWPT were nearly equal, while RVS V6RWPT was $32.0( \pm 9.5) \mathrm{ms}$ longer. The ROC curve analysis indicated delta V6RWPT > 12 ms as diagnostic of lack of HB capture (specificity of $99.1 \%$ and sensitivity of 100\%). A blinded observer correctly diagnosed 96.7\% (321/332) of ECGs using this criterion. Conclusions: We validated a novel criterion for HB capture that is based on the physiological left ventricular activation time as an individualized reference. HB capture can be diagnosed when paced V6RWPT does not exceed the value obtained during native conduction by more than $12 \mathrm{~ms}$, while longer paced V6RWPT indicates RVS capture.
\end{abstract}

\section{Hosted file}

Individualized HB diagnosis FIN 2805 2021.doc available at https://authorea.com/users/ 422495/articles/528208-novel-approach-to-diagnosis-of-his-bundle-capture-using-

individualized-left-ventricular-lateral-wall-activation-time-as-reference 